\title{
Educación y salud: dos campos de intervención, un interés común*
}

\section{Revista Colombiana \\ Artículos temáticos $\quad \begin{aligned} & \text { de Educación, N. }{ }^{\circ} 65 . \\ & \text { Segundo semestre de 2013, }\end{aligned}$ \\ Bogotá, Colombia.}

//Education and health: two fields
of intervention, a common interest

//Educação e saúde: dois campos

de intervenção, um interesse comum

Recibido: 28/06/2013 Evaluado: 08/10/2013

\section{Ana Maritza Gómez Ochoa**}

\begin{tabular}{l|l}
$*$ & Este trabajo se inscribe dentro de las reflexiones del grupo de investigación de Historia de las Prácticas Pedagógicas en \\
Colombia con énfasis en la Historia de la Educación, Pedagogía y Educación comparada de la universidad Pedagógica \\
Nacional. \\
Doctoranda en Educación de la Universidad Pedagógica Nacional de Colombia, Profesora de la facultad de Enfermería \\
de la Universidad Nacional de Colombia. Correo electrónico: amgomez@unal.edu.co
\end{tabular}

\section{Resumen}

El presente escrito indaga por la educación y la salud como dos campos que han compartido un interés común de intervención de la sociedad. Se parte de la afirmación de que, para la primera y segunda mitad del siglo XX, la medicina normalizó comportamientos y maneras de pensar, y la pedagogía hizo lo suyo tomando el cuerpo como objeto para disciplinar y organizar en el nuevo orden social.

La discusión comienza con la revisión del campo de la pedagogía en Colombia y sus diferentes concepciones para luego centrar la pregunta en las prácticas pedagógicas y cómo éstas funciona en el campo médico y dentro del discurso de la prevención y promoción de la salud. Nuevas modalidades discursivas emergen para la segunda mitad del siglo XX como la cultura de salud, conducida por una educación en salud, prácticas discursivas que finalmente terminan contribuyendo con una particular forma de gobierno de la población.

\section{Abstract}

This paper explores education and health as two fields that have tended to share a common interest in terms of intervention in society: for the first and second halves of the twentieth century, medicine normalized behaviors and ways of thinking, and pedagogy did the same, taking the body as an object to discipline and organize in the new social order.

Methods, techniques and procedures have been used as strategies which, organized in pedagogical practices, shaped the new citizen in organizational representations which claimed modernity. The discussion begins with a review of the field of pedagogy according to different Colombian visions. Then, it focuses the question of pedagogical practice in the discussion of modernity and how it is applied in the medical field within the discourse of prevention and promotion of health. New forms of discourse emerge in the second half of the twentieth century as a culture of health which, being conducted by health education, it is appropriate as a form of governmentality.

\section{Resumo}

O presente escrito indaga pela educação e a saúde como dois campos que compartilham um interesse comum na intervenção da sociedade. O ponto de partida

\section{Palabras Clave}

Práctica pedagógica, promoción y prevención de la salud, educación en salud, modernidad, gubernamentalidad.

\section{Keywords}

Pedagogical Practice, promotion and health prevention, health education, modernity, governmentality.

\section{Palavras chave}

Prática pedagógica, promoção e prevenção da saúde, educação em saúde, governamentalidade, modernidade. 
é a afirmação segundo a qual, na primeira e segunda metade do século XX, a medicina normalizou comportamentos e maneiras de pensar e a pedagogia fez o próprio tomando o corpo como alvo para disciplinar e organizar a nova ordem social. O texto faz uma revisão do campo da pedagogia na Colômbia e suas diferentes conceições para logo centrar a pergunta nas práticas pedagógicas e como estas funcionam no campo médico e dentro do discurso da prevenção e promoção da saúde. Novas modalidades discursivas emergem na segunda metade do século XX como a cultura da saúde, conduzida por uma educação em saúde, práticas discursivas que finalmente contribuem com uma forma particular de governo da população.

Un país se define no sólo por su actitud ante el futuro sino frente al pasado: su memoria no es menos reveladora que sus proyectos.

(Echeverry, 1997)

\section{Introducción}

La pedagogía y la medicina son quizás dominios diferentes en su estructura, posible expresión que designa dos lugares distintos de resistencia, de lucha, de realidad, de naturaleza, de extrañeza y de tensión; por sus características, una se ocupa de la educación y la otra de la salud. Pero ¿qué las hace particularmente cercanas? ¿Compañeras y aliadas silenciosas en la multiplicidad y la dispersión?

Responder a estas preguntas es la meta que se propone este escrito. Partimos de la tesis que plantea que la salud y la pedagogía han sido campos que desarrollan actividades intencionales; ellas configuran prácticas sociales y establecen relaciones con la enseñanza, con el aprendizaje y con el tipo de contenido a enseñar. En ese discurrir, la práctica pedagógica adquiere su sentido, convoca una relación susceptible de ser historiada desde aquellos sujetos participantes del discurso y sus funciones en torno a un saber.

"Aproximarse en la historia a la forma de lo dicho, en esta relación pedagogía y salud en nuestro país, es de alguna manera apropiarse de una historia, de un objeto de análisis institucionalizado 
con los sujetos que lo enuncian" (Álvarez, 2010a, cursivas mías). Para el campo de la salud, modalidades muy particulares de relaciones cobran visibilidad a partir de la preocupación por cambiar hábitos y costumbres, en tanto que, para el campo de la pedagogía, la preocupación es la construcción de intersubjetividades a partir del moldeamiento de cuerpos y mentes; estos elementos constituyen puntos de cercanía en esta relación (pedagogía y salud) para erigir un modo de ser y pensar en la sociedad colombiana.

Para este propósito, la pedagogía, en sus múltiples aristas, se constituyó, a la vez, en la condición de posibilidad y objeto para el nuevo reordenamiento social y cultural demandado por las sociedades modernas; nuevos significados para la educación, la higiene, la nutrición y la preocupación por el cuerpo se conformaron como fuentes de sabiduría que, apoyados en la ciencia y la tecnología, implementaron efectos de verdad en su discurso durante la segunda mitad del siglo XX.

Álvarez (2010b), en sus estudios sobre la escuela, el maestro y la configuración de la pedagogía, la presenta como el saber que da cuenta de un nuevo modo de ser de la época, abriendo nuevas preguntas y planteamientos a la modernidad, a partir de los cuales conforman nuevos dispositivos pedagógicos para educar a la población más allá de la escuela. Para el autor, "este asunto tendría que orientar los modos de educación escolarizados", así como reconoce "que existen otros modos de educación que le piden a la pedagogía su orientación" (p.3).

Así, indagar por la relación entre pedagogía y salud requiere realizar unas apuestas conceptuales cuya mirada dé cuenta de estos dos asuntos; aquí, la noción de práctica pedagógica cobra sentido por cuanto implica considerar a la pedagogía no en sí misma, sino en una red de relaciones y finalidades que se salen del campo exclusivamente pedagógico para actuar en otros campos, como el de la salud, que también tiene que ver con una relación establecida entre la enseñanza y el aprendizaje.

En concordancia, las preguntas que se plantean para este trabajo son:

¿Qué límites, resistencias y tensiones se han fijado entre el campo de la pedagogía y el campo de la salud en la segunda mitad del siglo XX?

¿Qué tipo de relaciones se establecieron entre prácticas pedagógicas y prácticas de educación en salud en la segunda mitad del siglo XX?

¿Cuáles fueron las condiciones de posibilidad que permiten pensar la relación pedagogía y salud en la segunda mitad del siglo XX?

Para lograr una aproximación de respuesta a estos cuestionamientos, se indagará, en principio, por las diferentes posiciones y discusiones planteadas desde la pedagogía por autores colombianos, tanto en el campo conceptual como pedagógico, para luego preguntar por la práctica pedagógica en el debate de la modernidad y la fuerza que esta 
tuvo para el campo médico en la segunda mitad del siglo XX. Posterior a esto, se hace el análisis de esta relación pedagogía y salud, tomado desde la perspectiva de la salud como comportamiento y el papel de la educación en salud como una forma de gubernamentalidad.

\section{Campo conceptual de la pedagogía}

En los últimos cincuenta años en Colombia, el debate pedagógico ha enfocado gran parte de su discusión en la reconfiguración de un campo conceptual que admita un estatus propio a la pedagogía; su preocupación central: el lugar de la escuela, el maestro y la pedagogía; recuperar la voz del maestro y su autonomía, formó parte de los objetivos. Sin embargo, en medio de la divergencia, dispersión y segregación entre educación y pedagogía, se plantearon claras diferencias entre ellos.

De una parte, el campo conceptual de la educación fue un asunto estudiado en Colombia por Mario Díaz, quien, a partir de los planteamientos de Bourdieu sobre campo ${ }^{1}$ y habitus ${ }^{2}$, tejió un entramado teórico aplicado al campo educativo; aquí los agentes y las instituciones crean un campo de fuerza y de lucha por un capital simbólico representado en diversas formas como económico, cultural, político o social, a partir del cual construyen reglas de juego dentro del campo, así como jerarquías, disputas y fronteras entre ellos; de ahí que diferentes posiciones y oposiciones determinan la estructura específica del campo en un momento histórico dado.

Sustentado en esta misma línea de reflexión, el campo conceptual de la pedagogía (CCP), planteado por Alberto Echeverry (2009), parte de la tesis según la cual "la pedagogía, más que un discurso ideológico, tiene la potencia suficiente para construir un campo de producción conceptual", no obstante "ser un campo de proliferación de conceptos y un sinnúmero de disciplinas que la sostienen", según argumenta Díaz (1998); para Echeverry (2009) se convierte en fortaleza: "la multiplicidad, en ciertas

1 "La estructura del campo es un estado de relación de fuerzas entre los agentes o las instituciones implicadas en la lucha, o, si se prefiere así, de la distribución del capital específico que, acumulado en el curso de las luchas anteriores, orienta las estrategias ulteriores" (Bourdieu, 2000, p. 113).

2 Habitus entendido como "sistema de disposiciones en vista de la práctica, constituye el fundamento objetivo de conductas regulares y, por lo mismo, de la regularidad de las conductas. Y podemos prever las prácticas [...] precisamente porque el habitus es aquello que hace que los agentes dotados del mismo se comporten de cierta manera en ciertas circunstancias" (Bourdieu, 1987;40). 
circunstancias, funciona como un obstáculo; en otras, potencia y enriquece el funcionamiento de la pedagogía, ya no como disciplina, sino como campo" (p.12).

En el CCP, se interroga por la pedagogía y el maestro desde la pedagogía misma, esta última desplegada a partir de la "proliferación" y la "multiplicidad"; como lo afirma Echeverry (2009), "la pedagogía, antes que un arte o un hacer, es una lectura del mundo para ser entregado a los niños y jóvenes" (p. 13).

En este sentido, el CCP, proclive a ser investigado en sus luchas y tensiones con la enseñanza, el maestro y el método, se pone hoy en tensión con otros campos y disciplinas. Por lo menos dos tendencias fueron establecidas para el campo pedagógico, las cuales el grupo de Historia de la Práctica Pedagógica en Colombia $(\mathrm{GHPP})^{3}$ describe en los siguientes términos: "hay quienes hablan de la contribución al campo conceptual de la pedagogía, y quienes prefieren hablar del campo de saber pedagógico" $^{\prime 4}$ (Álvarez, 2010c, p. 2);

3 El grupo Historia de la Práctica Pedagógica en Colombia, fundado en 1978 por la profesora Olga Lucía Zuluaga, en cabeza del grupo, y los profesores Jesús Alberto Echeverri Sánchez, Humberto Quiceno Castrillón, Alberto Martínez Boom, Estella Restrepo. Recuperado de www. cienciagora.com.co/galeria_de_cientificos/ciencias-de-la-educacion-154/olga-zuluaga/315.html

4 Para mayor claridad, se puede consultar en el documento: Del grupo de Historia de las Prácticas Pedagógicas en Colombia: aportes para un debate sobre el estatuto teórico de la pedagogía. Referencia del profesor Alejandro Álvarez Gallego (2010). Profesor Universidad Pedagógica Nacional. Miembro del grupo de Historia de la Práctica Pedagógica. la diferencia radica en que la primera tendencia se interesa por estudiar la pedagogía como campo disciplinar dotado de un cuerpo conceptual teórico y la segunda, por estudiar los enunciados que han configurado el campo, con lo que se sale de un marco disciplinar rígido en su estructura y se sitúa en la heterogeneidad y dispersión para mirar de otro modo sus posibles tensiones y resistencias.

En este orden de ideas, se puede precisar que en Colombia comenzó la discusión de la pedagogía como campo hacia finales de los años setenta, ligado a referentes formativos y teorizaciones pedagógicas tomadas de otras disciplinas como la psicología y la sociología, entre otras, para preguntar por el maestro y su enseñanza en el mundo contemporáneo; reafirmado en una lectura pedagógica a partir de los clásicos como Vives, Comenio, Herbart, Kant, Pestalozzi, Claparede, Rousseau, Montessori, Freinet y Piaget, entre otros. Se intentó, más que llegar a una verdad, analizar, desde el lente de la modernidad en medio de la crisis, cuál era la función de la pedagogía en el intento por recobrar su propia voz frente a la crisis de la civilización y la cultura, y cómo la pedagogía, al ser sustituida por los dispositivos de la tecnología, demarcando claramente la distancia entre tradición y modernidad, obligaría a mirarla desde el exterior y pensar en su reconceptualización.

Varios grupos de trabajo de pedagogos se venían conformando en el 
país para la segunda mitad del siglo XX; estos impactaron en la visión pedagógica y fomentaron propuestas innovadoras que delimitaron nuevas miradas a la pedagogía, como estatuto epistemológico propio que se instauró para desprenderse de las otras disciplinas a las cuales había estado sometida. En el debate por el maestro, la enseñanza incluía el método y la escuela como el lugar de la acción pedagógica.

Los aportes del GHPP al campo conceptual de la pedagogía se relacionaron en considerar a la pedagogía como saber y como práctica; la noción de práctica pedagógica propuesta por el grupo permitió, según la profesora Zuluaga, "historiar a la pedagogía en tanto discurso y práctica acerca de la enseñanza; allí se contempló las prácticas de enseñanza en diferentes espacios sociales mediante elementos del saber pedagógico" (2005, p. 23).

Para el grupo, la orientación metodológica de la arqueología y genealogía marcó el derrotero por el cual se podría ir más allá de los conceptos y las prácticas de su propio campo; los referentes teórico-metodológicos planteados por el filósofo francés Michel Foucault posibilitaron el análisis para encontrar distintas relaciones con otras prácticas y disciplinas que, en todo caso, se conectaban con la pedagogía y su objeto: la enseñanza.

En esta perspectiva, Zuluaga (2005) anuncia:

Dotó al grupo de Historia de la Práctica Pedagógica en Colombia de un lente para entrar en relación con destacados pensadores de la pedagogía y para observar en los documentos que componen el archivo pedagógica del país el cruce de conceptos y prácticas (p. 16).

Las nociones que dieron piso al proyecto de investigación del grupo fueron, en principio, las de saber y práctica discursiva de Foucault, sobre las cuales elaboraron las de saber pedagógico y práctica pedagógica; de entrada, esta relación les permitió describir la pedagogía como práctica discursiva y entender que no existe saber sin una práctica, en palabras de Zuluaga (2005):"no se puede entender la práctica sin el saber y viceversa, es decir, práctica y saber son al mismo tiempo objeto y condición de posibilidad" (p. 20).

Aproximarse a una historia de las prácticas discursivas implicó para el GHPP hacer una revisión documental exhaustiva de los objetos y saberes institucionalizados y los sujetos implicados en el entramado de su funcionalidad enunciativa, a partir de sus acuerdos, tensiones y rupturas, para rastrear en los discursos de 
la época de estudio, así como hacia su exterioridad, esta compleja red de relaciones y percibir en sus memorias los enunciados que traspasaron la época e influyeron en la circulación de saberes.

Con posterioridad, hacia los años ochenta, se conformó el grupo Federici ${ }^{5}$, el cual se nutrió de los argumentos de la teoría crítica de Jürgen Habermas, inscrita en el contexto de la tradición filosófica cultivada por los miembros de la denominada Escuela de Fráncfort, y en el pensamiento de Hans-Georg Gadamer, para hacer sus aportes al pensamiento pedagógico colombiano, desde dos frentes: el primero reconoció la enseñanza como una acción comunicativa de los maestros y abogó por la construcción de alternativas didácticas para lograr competencias especializadas en la enseñanza de las ciencias; el segundo se dirigió a la denuncia y la

5 Carlo Federici (1906-2005). En los años ochenta, el maestro Federici organizó varios seminarios en la Facultad de Ciencias Humanas de la Universidad Nacional de Colombia para discutir temas relacionados con pedagogía. Estas reuniones originaron la primera generación de llamado Grupo Federici, del cual hicieron parte reconocidos intelectuales como Antanas Mockus, Jorge Charum, Berenice Guerrero, María Clemencia Castro, Carlos Augusto Hernández y José Granés, entre muchos otros. "Federici comenzó rompiendo los rígidos esquemas pedagógicos y los rituales formalistas, usuales en las prácticas educativas del momento, en tanto que inicia discusiones alrededor de la educación en las ciencias y las matemáticas, discusión de las reformas curriculares para la escuela básica y media, y trabaja en un proyecto sobre la formación de una actitud científica en el niño a través de la enseñanza de las ciencias naturales y la matemática, entre otros". Recuperado de www.unal.edu.co. Grupo Federici. crítica de un modelo curricular con enfoque taylorista y llamó la atención de los maestros sobre el peligro de un diseño instruccional, que ponía en riesgo su práctica pedagógica y su identidad de maestro (Tamayo, 2006, p. 107).

Parte de las orientaciones del grupo se dirigió hacia la crítica de la reforma curricular que en su momento se había iniciado, cuyas bases cientificistas y positivistas se legitimaron bajo la categoría tecnología educativa como una manera particular de asumir el aprendizaje y el conocimiento en general, por eso las fuertes críticas se dieron en el orden de la estandarización de los contenidos, que convertían al maestro en simple ejecutor de guías y planes de enseñanza (Gómez, 1989, p. 17).

Otros aspectos estudiados por este grupo fueron los referentes a la calidad de la educación, en cuanto conocimiento y comunicación dentro y fuera de la escuela. La medición de la educación por resultados, como la propuesta por el Icfes, fue fuertemente cuestionada: "por ello el trabajo diario, así como sus efectos más importantes a largo plazo, pueden juzgarse más que por la cantidad o novedad de los contenidos, por el grado de acceso al dominio de esas formas de comunicación y de conocimiento" (Mockus y Charum, 1986, p. 22).

Hay que resaltar aquí un estudio del GHPP, realizado por Martínez, Noguera y Castro (1994), que describe las primeras experiencias de educación fundamental en Colombia hacia finales de los años cuarenta; 
en esta reflexión, se describe el periodo instruccionista, marcado por varios acontecimientos como: "la misión Currie en 1949, que planteó la articulación de desarrollo con educación, y posteriormente en 1956 con la misión Lebret, que la hizo realidad" (p. 92); este modelo de desarrollo implicó urbanización de la población, escolarización y normalización. Triada estudiada ampliamente por estos autores y en general por el GHPP.

La apertura de las escuela radiofónicas hacia este periodo en Colombia se presenta en este estudio como el acontecimiento que marcó el comienzo de la utilización masiva de los medios de comunicación para llevar programas de alfabetización a sectores concretos; para ello, la utilización de cartillas, organización de bibliotecas y utilización de guías de instrucción fueron los medios utilizados para el entrenamiento de maestros, educación en salud, educación en prisiones y educación del personal militar.

En este orden de ideas, el tema de la enseñanza, fuertemente debatida en torno a al saber y a la autonomía del maestro, sería una de las cuestiones que posibilitó a los pedagogos y grupos de investigación iniciar los debates de los nuevos escenarios que planteaba la pedagogía, en concordancia con la modernidad y el desarrollo de las apuestas políticas y económicas del momento. Claramente advierten los autores que, para esta época, las experiencias educativas al margen de la escuela hacen su emergencia en los sectores populares con un elemento particular de programación y diseño instruccional, sin desconocer que "a finales de la década de los cuarenta, se inicia un proceso de generalización de la educación primaria gratuita y obligatoria en todos los países del tercer mundo". (Martínez, Noguera y Castro, 1994, p. 25).

Por su parte, el profesor Quiceno aborda la pedagogía como acontecimiento, pero advierte sobre el cuidado con el que se deben ubicar los procesos que la afectan desde su exterioridad, los cuales intervienen y se truncan con los procesos cerrados en la medida en que se piense la organización de la información y los aspectos de la subjetividad. Al respecto, afirma:

Su objeto (la pedagogía) ya no puede ser el hombre y su fundamento la naturaleza o la sociedad. La pedagogía debe plantearse seriamente los problemas de la vida, de la comunicación y de la información del hombre como ser viviente en relación con esta red de sistemas y sus efectos sobre la cultura y las formas de poder-saber (Quiceno, 1998, p. 146). 
Otros aportes que hicieron eco en los debates pedagógicos fueron los realizados por los grupos de educación popular ${ }^{6}$, que, a partir de sus trabajos comunitarios de educación de adultos y educación popular, marcaron la relación escuela-comunidad ligados a los movimientos sociales $y$, en oposición a la educación formal, su voz se desplegó en las apuestas políticas y pedagógicas del país; enraizado en las propuestas de Paulo Freire, convocó para el país, y en general para toda América Latina, a las pedagogías de la liberación y las pedagogías sociales, enmarcado en las corrientes críticas y emancipadoras.

El tema del conflicto, la violencia y la paz generó, por parte de estos grupos, un asunto a tratar en sus agendas de trabajo (Muñoz, 2004, p. 5); como propuesta pedagógica, planteó el diálogo de saberes y el diálogo cultural. Su relación con el campo de la pedagogía en continua tensión y disputa, por cuanto no se construyó un edificio conceptual, los llevó a ocuparse de la enseñanza y la reconstrucción del pensamiento pedagógico de los educadores.

Lo planteado hasta aquí visibiliza, para la segunda mitad del siglo $\mathrm{XX}$, las diferentes visiones y posiciones pedagógicas procedentes de heterogéneas orientaciones de trabajo, las cuales tienen como punto de partida la tensión entre pedagogía, escuela y maestro; lo que se daría

6 El CINEP (Centro de Investigación y Educación Popular), Dimensión Educativa y el CEPECS (Centro de Promoción Ecuménica y Comunicación Social), entre otros. a partir de su proliferación y multiplicidad, según palabras del profesor Alberto Echeverry. por otro lado, la preocupación de otros grupos de trabajo por dar un estatus propio a la pedagogía en Colombia, como campo de saber y de poder, a partir de la conceptualización y reconceptualización del CCE y CCP, que en medio de la crisis educativa establece una relación directa con la modernidad, lo cual instaura, de una parte, la discusión sobre su cientificidad y, de otra, como asunto político, en cuanto dispositivo para la regulación y homogenización social. Finalmente, están los aportes del movimiento popular como movimiento social y emancipador en un diálogo con la cultura y la sociedad.

Se puede concluir, para este apartado, que el Campo Conceptual de la Pedagogía abre la posibilidad de encuentro o desencuentro entre la teoría frente a sus problemáticas como recurso para discutir sus procesos de institucionalización emergentes y constituyentes de nuevos sujetos y saberes al campo pedagógico. Para Álvarez (2010c;), allí "se toma distancia de otros acontecimientos, se excluyen verdades, en fin. Inscribiendo estas tradiciones en un campo conceptual, se producen préstamos e intercambios que podrían enriquecer a su vez a cada una de ellas" (p12).

Desde esta perspectiva, las orientaciones del campo del saber pedagógico permiten la articulación y acercamiento con otros saberes y prácticas que son consideradas 
significativas, y aunque no sigan las rigurosidades académicas, se apropian de un lenguaje y una cultura para problematizar mas allá de la escuela; estos campos se relacionan con la salud, la política, la economía, etc.: "el Campo de Saber Pedagógico incluiría todo este tipo de discursos, con sus regímenes de enunciación propios y sus reglas de funcionamiento que desbordan y cuestionan la escuela" (Álvarez, 2010c, p. 4). Estos dos campos serían los que permitirían reconocer, históricamente, la manera como se constituyó cada uno de estos paradigmas y encontrar en su devenir sus filiaciones, sus especificidades, sus conceptos y sus problemáticas.

Un trabajo de largo aliento, con mucho material de análisis, que nos invita a trabajar en dos frentes: a pensar el problema de la reconfiguración de una cultura pedagógica, con lo que abre la posibilidad de nuevas formas de diálogo con otros saberes y disciplinas, que, para el caso que nos ocupa, como es el campo de la salud, retoman para sí a la pedagogía como objeto y condición de posibilidad para argumentar sus propias posturas.

Desde esta mirada, el campo de la pedagogía muestra cómo en cada dominio discursivo se discute su cientificidad, sus teorías y conceptos, así como se institucionalizan procesos y emergen sujetos y oficios. Cabe entonces, a partir de las conceptualizaciones arriba señaladas de educación y pedagogía, comenzar a dialogar con la práctica pedagógica como un campo dinámico en la discusión, por cuanto se relaciona con los saberes institucionalizados, enmarcados desde un saber-poder e incrustado en los debates de la modernidad, para ir comprendiendo su fuerza como una forma de gobierno de sí y de los otros, que tomó forma a través del discurso de la salud representado en el campo de la medicina.

\section{La práctica pedagógica en el debate sobre la modernidad}

Examinar los debates actuales sobre modernidad y modernización educativa es sin duda el primer paso para encontrar el lugar que ocupó la práctica pedagógica desde sus tensiones y transformaciones como forma reflexiva de problematizar los diferentes caminos que trazaron los investigadores, leídos desde su inteligibilidad e interrogantes en los nuevos escenarios de conflicto y contradicción que ofreció la modernidad en la segunda mitad del siglo XX. 
La modernidad es un concepto articulador de las ciencias sociales en general y de la educación en particular, ésta se determina a partir de la continuidad y emergencia de acontecimientos marcados como hechos históricos que definieron cada época, creando un marco de interpretación que vendría a convertirse en las condiciones de posibilidad para cambiar las reglas del juego de la vida social Ilevadas hasta cierto momento y en un espacio determinado.

Autores como Hardt y Negri (2000) hablan de la modernidad a partir de la declinación de la soberanía de los estados-nación, "lo que no significó la declinación de la soberanía, sino un giro acaecido de los mecanismos regulatorios, tomando nuevas formas, más allá de los organismos nacionales" (p. 34).

En este análisis de modernidad para la segunda mitad del siglo XX, según Hardt y Negri (2000): "no es posible desligar el papel de la educación con la desnacionalización del Estado para dar paso a la globalización y el advenimiento del imperio y la multitud que afectaron la naturaleza del saber". La modernización tiende cada vez más a ser totailizante, por cuanto su producción abarca lo económico, político, social y cultural y su principal finalidad es la creación de riqueza para configurar nuevas formas de gobernamiento a partir de cambios productivos que obligan a la configuración de nuevos paradigmas.
El Imperio no establece centro territorial de poder, y no se basa en fronteras fijas o barreras. Es un aparato de mando descentrado y desterritorializado que incorpora progresivamente a todo el reino global dentro de sus fronteras abiertas y expansivas. El Imperio maneja identidades híbridas, jerarquías flexibles e intercambios plurales por medio de redes moduladoras de comando. Los diferentes colores del mapa imperialista del mundo se han unido y fundido en el arco iris imperial global (p. 14).

Democracia e industrialización, para Stramiello (2010), "fueron la piedra angular del desarrollo económico-social, en el que la educación cumplía un rol decisivo. Se esperaba que la industrialización ayudara a la liberación política y económica de las naciones latinoamericanas" (p. 396). Las reformas mantenían vínculos cercanos con la economía, mientras los aspectos sociales y culturales adquirieron un carácter instrumental; por tanto, para la autora: "la educación se presentaba como el instrumento para combatir el desempleo, para impulsar los avances científicos y tecnológicos, para defender la democracia y para lograr el desarrollo social" (p. 399). 
Así, Stramiello (2010) nos documenta:

En América Latina, la discusión de los sistemas educativos fue una prioridad y el tema de desarrollo económico se convirtió en el eje principal para el desarrollo de la nación; llegar a la población fue un asunto fundamental. En este sentido aumentó el número de alfabetos, aunque el índice de analfabetismo continuó altísimo, sobre todo en las zonas rurales" (p. 397).

Para Gajardo (1999), las políticas de reformas de los noventa se desarrollaron en torno a cuatro ejes principales: gestión, calidad y equidad, perfeccionamiento docente y financiamiento; las propuestas políticas se sustentaban en los enunciados de democracia y economía, restándole menor intervención del Estado y priorizando en la economía de libre mercado.

Estos cambios en el consumo económico, de producción y en la estructura misma de la interacción social que ha planteado la modernidad han incidido de manera directa en el cambio de la visión pedagógica, que hoy reclama también por una modernidad, por cuanto plantea nuevos desafíos y logros, que para Gajardo (2009) tienen que ver sobre todo con el aumento de la cobertura de la enseñanza, centrar la atención a la calidad de la educación mediante la medición de resultados e integrar la salud escolar en los currículos. En torno a esto, Ratinoff, (1994) ${ }^{7}$ nos anuncia: "las instituciones docentes tenían que adoptar una pedagogía pluralista, reemplazar el formalismo académico y la homogeneidad cultural y llegar a la aceptación por la diversidad"(p. 17).

Aquí, la pedagogía pensada y operada a partir del concepto de desarrollo y modernidad emergió como la condición de posibilidad que permitió impulsar y convertirse en aliada de las

7 Luis Ratinoff (1994, pp. 15-98) distingue cuatro momentos estratégicos en el análisis de la evolución de las retóricas educativas: 1. El nacionalismo educativo predomina poco antes de la Segunda Guerra Mundial y enfatiza la creación social de valor a través de los esfuerzos para construir la nación; 2. El pluralismo educativo, que dura el corto interludio del final de la guerra y pocos años antes de la Guerra Fría, acentúa la importancia de la escuela en la formación de sociedades abiertas y democráticas; 3 . El culto al mérito que expresa las preocupaciones del largo periodo de la confrontación ideológica y destaca el papel estratégico de la escuela en la formación de los cuadros dirigentes en sociedades estratificadas; y el 4. Las retóricas del capital humano que están profundamente asociadas al periodo del "final de la historia", con el énfasis puesto en la globalización de los procesos y en la reducción de los compromisos político nacionales, que desvía la atención de los propósitos a la organización de los medios educativos, puesto que el nuevo papel de la escuela es programar la productividad de los individuos. La crisis de la educación: el papel de las retóricas y el papel de las reformas. 
nuevas propuestas desarrollistas del campo educativo, pedagógico y de la salud. Alternamente a los hechos de la modernidad, la pedagogía y su práctica comenzaron a ser cuestionadas e interrogadas a partir de un afán civilizatorio: "es evidente la vocación civilizadora que ha tenido la pedagogía en el país, vocación que en el presente siglo se ha vivido como un afán racionalizador y modernizador" (Sáenz, 1997, p. 113).

Vásquez (2003), por su parte, consideraba que la educación de la mano de la investigación mejoraría el ejercicio de la ciencia y la tecnología y promovería mayor independencia y autonomía; sin embargo, se priorizaría en la educación básica como una exigencia del Banco Mundial. Por tanto, la agenda de modernización de la educación fijó como objetivos: "mejorar la calidad del sistema educativo, elevar la escolaridad de la población, descentralizar la educación y fortalecer la participación de la sociedad en el quehacer educativo" (p. 5).

Para el caso de Colombia, desde el siglo XIX, la instrucción pública adquiere un carácter social, que, según Martínez Noguera y castro (2003, p. 240), "esta dimensión social estaba subyugada al ejercicio de un poder que, más que ejercerlo, estaba garantizando la continuidad de los regímenes guardianes de la democracia, proyectando así más un proyecto político que educativo"; según los autores, un hito histórico marcado por la Segunda Guerra Mundial fue el acelerado proceso de desarrollo económico, social y cultural manifiesto por el término de desarrollo, que se convirtió en una estrategia generada a partir de 1945 en los países industrializados, creando un nuevo campo delimitado por la noción de subdesarrollo y por el despliegue de nuevas formas de ejercicio del poder.

Durante la segunda mitad del siglo XX, la educación en Colombia pasa de un modelo disciplinario y regenerativo, marcado por el modelo higienista, a un proceso democrático con una alta tendencia a la escolarización y curricularización masiva; el campo de la salud para los dos periodos (primera y segunda mitad del siglo XX) se encuentra articulado al modelo educativo de diferentes formas que se describirán más adelante, pero en la perspectiva de metas planteadas por nuevas estrategias de desarrollo en la que, además de la preocupación por la enseñanza de las letras y las matemáticas, se encontraba también la preocupación por la enseñanza de la salud.

En esta perspectiva, se planteó la educación como un proceso abierto de difusión de valores apoyados en la gobernabilidad y democracia, su función principal: contribuir a la construcción, modernización e integración de la nación; paz, salud y educación constituyeron el trípode que delimitaría la finalidad de la acción pedagógica.

De esta manera, la práctica pedagógica se apropió de nuevas categorías y se reconceptualizó como saber y como campo en un 
horizonte de producción de nuevos conceptos y prácticas en medio de la crisis de la modernidad, que, en palabras del profesor Echeverry, se produjo a partir de la multiplicidad y proliferación. La tensión entre lo tradicional y lo moderno se develó en el debilitamiento de la enseñanza, la pregunta por el papel del maestro y la relación escuela-sociedad, que, afiliado al campo de la salud, instituyeron una complicidad para determinar el nuevo tipo de hombre a formar; la tecnología, la circulación del capital y la pérdida de fronteras definieron, en gran parte, a la sociedad contemporánea, lo cual, aliado a la pedagogía, sirvió de base y de estrategia para lograr intervenir la sociedad ahora moderna.

\section{Noción de práctica pedagógica: un campo de saber y de poder más allá de la escuela}

En la primera parte de este escrito se enunció cómo fue emergiendo una nueva discursividad en la segunda mitad del siglo $\mathrm{XX}$, que para el caso colombiano, se planteó a partir de nuevas visibilidades de la pedagogía, las cuales pasaron a considerarla en tanto práctica despojada de todo aquello "tradicional", para instaurar nuevos retos que se fueron construyendo con los discursos procedentes del marco global de desarrollo y contribuyó a un nuevo modo de ser y pensar en la sociedad.

Por eso, la pretensión en este apartado es preguntar por los diferentes conceptos y orientaciones que se han formulado alrededor de las prácticas pedagógicas en medio de la proliferación y multiplicidad, que hoy convoca otros campos y frentes de trabajo, las cuales se han tensionado tanto en el campo del saber pedagógico como fuera de él para determinar una nueva discursividad, que también reclama como espacio correlativo el campo de la salud.

Noguera (2013) nos señala "que el término pedagogía es usado para referir la práctica de conducir y orientar la conducta de otros"; bajo estos enunciados, las prácticas pedagógicas se expresaron como una forma de conducir las conductas de los individuos, que para Díaz (1990) se instituyó como dispositivo de regulación de discursos/significados, prácticas/formas y transmisión de significados; tales prácticas operadas para la modernidad, se materializaron en planes, programas, técnicas, que tuvieron como finalidad la conversión del sujeto; aquí la práctica 
pedagógica se abrió paso de una escuela de encierro a una escuela abierta y de conocimiento.

Para la década de los ochenta y noventa del siglo XX, autores como Sirotnik (1989), Escudero (1984) y De Miguel (1990) refirieron cambios de renovación en la educación; consideraron a la escuela como el lugar por excelencia para el cambio, donde, a partir de cada realidad concreta como institucionalidad, se deberían elaborar planes de formación para alumnos y maestros.

Otros autores ampliaron la intencionalidad de la práctica pedagógica no solo en la escuela y el maestro, sino que pasó al orden social; diferentes reflexiones reconocen a la práctica pedagógica del lado de los procesos y sistemas en cuanto proceso intencional que, según Huberman (1994):

Se sitúa en un sistema deliberado, consciente $y$ participativo para mejorar desempeños y resultados, estimular el desarrollo de la renovación en campos académicos, profesionales o laborales y formar el espíritu de compromiso de cada persona con la sociedad y particularmente para la comunidad en la cual se desenvuelve (p.11).

La práctica pedagógica como escenario histórico registró objetos de saber, nociones, conceptos que dieron cuenta de la pedagogía, la educación, el currículo, la didácti$\mathrm{ca}$, en funciones de su materialidad, conceptos, formas de ser de una época, se ligaron no solo a la escuela, sino a otras institucionalidades, como el hospital, la fábrica, la familia y otras subjetividades como la enfermera, el médico, a los que, agrupados, se les confiere una pluralidad que a la vez constituyen en especificidad cuando entran en acción con una sociedad. La historia de la práctica pedagógica para la profesora Zuluaga:

Se efectúa sobre su propia discursividad, sobre el propio régimen al cual estuvo sujeta, no necesita interrogar otras prácticas para conferirse identidad, porque ella está inmersa entre otras prácticas sin que por ello se borre su especificidad, y tiene así sus límites bien demarcados por el saber pedagógico y por la práctica de ese saber en nuestra sociedad (1999, p. 15).

Hasta aquí, la discusión tiene varias vertientes: una se concentra en el maestro comunicador y enseñante de lo ya dicho en relación con el conocimiento y suposición como sujeto autónomo o no, supeditado a una producción de significados desde el poder-control, en la que se cuestiona la autonomía del maestro. Otra pasa al orden social, la práctica pedagógica se legitima en otros escenarios que plantean también el problema 
de la enseñanza y su relación con la sociedad, la cultura con sus valores, objetos, experiencias y formas de comunicación; estos procedimientos se relacionan con el control, relaciones de fuerza, a partir de las cuales caracterizan prácticas con sus modalidades enunciativas que se concretizan en niveles de autoridad.

La potencia y fuerza de los discursos contemporáneos se perciben como la emergencia de nuevos lenguajes que se instituyen en una práctica discursiva ${ }^{8}$, ligada a un sujeto pedagógico con la intención de orientarlo a nuevas alternativas propuestas por la modernidad para la formación de un sujeto que responda a la triada capital humano, mercado y producción. El papel de la pedagogía en este sentido organiza, explica, regula y pone a funcionar la gramática de la re-contextualización ${ }^{9}$ en una práctica reconstructiva susceptible de ser teorizada y enseñada, pero con una finalidad muy clara: conducir a los individuos.

\section{La fuerza de la práctica pedagógica en la mirada médica}

Siguiendo la misma línea de análisis, el campo de la medicina en Colombia asumió una activa participación en el movimiento modernizador, definió políticas con altas tendencias desarrollistas enmarcadas en la formación de un sujeto higiénico para la primera mitad del siglo XX y un sujeto saludable para la segunda mitad del siglo XX ; su amplia intervención abarcó la promoción y protección de la salud, lo cual se orientó a la infancia, la mujer y el hombre como fuerza de trabajo y capital social y la familia como institucionalidad concreta.

La medicina en cuanto campo de saber y de poder se definió como unas reglas concretas de vida, justificadas a partir de un conocimiento científico y requirió del concurso de un conjunto de disciplinas y profesiones tales como: enfermería, nutrición y dietética, trabajo social, bioestadística, microbiología, química, antropología, bromatología, toxicología, economía política

8 La categoría de práctica discursiva, tal como ha sido propuesta por Foucault (1970, pp. 306-310), consiste en que "ningún discurso se da fuera del sistema de relaciones materiales que lo estructuran y lo constituyen (...) en toda formación discursiva se encuentra una relación específica entre ciencia y saber (...) La palabra práctica, designa la existencia objetiva y material de ciertas reglas dentro de las cuales el sujeto toma parte en el discurso. Sus efectos son analizados como posiciones del sujeto"

9 Recordemos que hacia los años setenta comenzó a abrirse paso la inquietud por la investigación sobre la pedagogía en Colombia. Un trabajo pionero fue el propuesto por el grupo de Historia de la Práctica Pedagógica en Colombia. 
y la sociología, entre otras, que, articuladas a las preocupaciones de los problemas contemporáneos, delimitaron un modo de vida para la población con una clara acción pedagógica.

Las enfermedades de la población se constituyeron en blanco del gobierno, que, para principios de siglo $X X$, se hallaban claramente clasificadas en endémicas y epidémicas ${ }^{10}$; el problema de la morbi-mortalidad se constituyó en la preocupación central de los gobiernos y poderes públicos. Estas epidemias, Ilamadas por Foucault (2000, pp. 220) "la muerte multiplicada", se volvieron endémicas, constituyendo una amenaza y una preocupación por la disminución de la fuerza de trabajo, la baja rentabilidad y el aumento de costos económicos, por cuanto decrecía la producción y aumentaba la demanda de atención a los enfermos.

El modelo teórico orientador basado en la teoría ecológica (1935), que incluyó el agente, huésped y medio ambiente como los factores interventores en los procesos de morbilidad, constituyeron el modelo epidemiológico unicausal predominante hasta los años sesenta. La medicina sustentada por la ciencia buscaba las causas de las enfermedades al tiempo que establecía y sancionaba a los portadores de enfermedades infecto-contagiosas, de

10 Las epidémicas son enfermedades que vienen de afuera, en oleadas y en poco tiempo pueden arrasar con la población. Las endémicas, por el contrario, conviven con la población humana y animal de un lugar. allí que las enfermedades establecieron un nivel de jerarquización; ignorancia y pobreza se unieron a la categoría de enfermedad, situación que se definía en gran parte a través de campañas educativas, donde la vigilancia, el control y el seguimiento conformaron la acción pedagógica para la intervención a la población. "El saber médico en Colombia, por su diversidad, sus desfases y controversias, formó un conjunto ecléctico y en cierta medida vanguardista, es decir, siempre con pretensiones de innovar o de estar a la altura de la modernidad" (Márquez, 2010, p. 10).

La higiene fundamentada en los debates científicos adquiere sentido para la pedagogía; esta se convierte en el elemento de la acción pedagógica y llega a constituirse en una acción combinada mediada por intereses similares: la medicalización de la sociedad de la mano de la escolarización. En tanto la higiene fue considerada fundamentalmente como restauración fisiológica, su aporte al progreso económico del país se hizo evidente. Los debates de la raza, la pobreza e ignorancia fueron asuntos centrales en las agendas de trabajo y propuestas médicas.

Se precisa aclarar que la relación educación-salud no fue un asunto emergente en la segunda mitad del siglo $\mathrm{XX}$; su visibilidad procede en los últimos treinta años del siglo XIX; el campo médico fue especialmente dinámico en este periodo; su producción científica acerca de la etiología, causa y propagación de 
las enfermedades ${ }^{11}$, así como en los conocimientos de la anatomía, fisiología y la clínica, permitió las condiciones materiales de existencia para que tomara un lugar en el orden social y se valiera de la educación como práctica y estrategia para producir modos de vida definidos. Para Noguera (2006), "el naciente proceso de industrialización de Colombia a comienzos del siglo XX requería una mano de obra sana y fuerte. La población fue considerada entonces como la principal riqueza con la cual contaba la nación".

Hacia finales de los años cuarenta, toma fuerza el concepto de salud pública, ésta se orienta a la preservación y perfeccionamiento de la salud individual y colectiva, incluyendo la prevención de las enfermedades; una particularidad para el país se vinculó en la búsqueda de la integración, jerarquización y mayor especialidad en los servicios de salud, para superar la visión tradicional sobre el control y erradicación de las enfermedades trasmisibles a través de la articulación de la medicina preventiva, curativa y de rehabilitación.

Posteriormente, hacia los años sesenta, se observó un desplazamiento de la concepción biológica de la salud, integrando la idea de salud como un factor de desarrollo, en palabras de Yepes (1990), "no es posible el desarrollo sin avances decisivos en el terreno de la salud; política económica y política de salud son vistas como elementos inseparables" (p. 40). El proceso biológico se miró como un hecho ligado a las condiciones que rodean la vida humana, y la epidemiología se vio abocada a cambiar el concepto de unicausalidad por el de multicausalidad; lo cual, al ser retomado por autores como Leavel y Clark (1976), entre los años cincuenta y sesenta, dio paso a la utilización del concepto de promoción de la salud, al desarrollar el modelo de historia natural de la enfermedad. Esta comporta tres niveles de prevención, en los cuales existen, por lo menos, cinco componentes distintos, dependiendo del grado de conocimiento de la historia natural ${ }^{12}$.

Estas nociones concedieron, para el caso colombiano, establecer una relación directa con la educación a través de acciones de tipo pedagógico implementadas en todos aquellos espacios

11 Se desplazan las concepciones hipocráticas sobre los orígenes de las enfermedades (miasmas, pestilencias, aires pútridos) para dar paso a los descubrimientos pasteurianos, que ponen de manifiesto la acción de los microorganismos en las enfermedades. Bacilos, virus, bacterias y gérmenes (Quevedo, 1994).

12 Prevención primaria abarca la promoción de la salud y la protección específica; prevención secundaria comprende el diagnóstico precoz y tratamiento oportuno y la prevención terciaria incluye la limitación del daño y la rehabilitación. 
donde fuese posible reunir la mayor cantidad de población posible, como los centros de salud, hospitales, escuelas, lugares de trabajo, la familia y la comunidad, que para Hernández (2002) "estuvieron enfocados principalmente a problemas de desnutrición, enfermedades gastrointestinales, inmuno-prevenibles y transmisibles, así como los relacionados con el medio ambiente y falta de accesibilidad a los servicios de salud, en especial, a los de atención primaria" (p. 167).

Hacia los años setenta, se habló de la reconceptualización del campo de la salud; esta nueva visión abarcó la relación salud-enfermedad-cuidado, articulada a condiciones de vida; este cambio, según Czeresnia (2009), "obedeció a la necesidad de controlar los costos desmedidamente crecientes de asistencia médica, que no correspondían a sus resultados significativos"; esta nueva salud pública, afirma la autora, "ocurre en el contexto de sociedades capitalistas neoliberales" (p. 43).

Para 1974, Marc Lalonde, ministro de Salud de Canadá, publica un documento oficial que propone la promoción de la salud como una estrategia y una política pública para un país; esta propuesta parte del compromiso de la gente en aquellos aspectos que determinan su bienestar; por tanto, esta estrategia pretendió vincular a la gente en el control de los principales determinantes de salud y acatamiento de la responsabilidad sobre su estilo de vida; en los países industrializados, el enfoque predominante fue el interés por los estilos de vida; de manera que esta nueva visión se asumió como estrategia, de allí la importancia de la educación para la salud como una forma de lograr las metas en salud (Gómez, 2004, p. 10).

En cuanto categoría apropiada promoción de la salud, el camino de la salud-enfermedad cobra nuevas visibilidades: por un lado, se enuncia a la promoción como comportamiento desde varios niveles (social, político, económico y cultural); y, por otro, la educación toma un carácter promotor y terapéutico, que, unido a la categoría de ciudadanía, adquiere mayor importancia el aspecto social y colectivo.

La promoción de la salud tuvo una trascendencia importante a finales de los años ochenta al emitirse el Acuerdo General (Carta de Ottawa de 1986), cuando la salud pública de enfoque comunitario y la promoción de la salud se constituyeron en una nueva concepción de salud pública (OMS); este giro conceptual, con nuevas categorías incluyentes como entornos, autocuidado, estilos de vida, vinculó la salud a las políticas de desarrollo del Plan Nacional en Colombia, y tomó fuerza la medicalización, desarrollo tecnológico, transición demográfica y cambio en el perfil nosológico, constituyendo un sistema amplio y globalizante a partir de la atención primaria en salud $^{13}$, enfatizando principalmente

\footnotetext{
13 "La atención primaria en salud involucra los siguientes aspectos para su desarrollo: la promoción y la prevención, dentro de los cuales se
} 
en promoción sin que desapareciera la prevención; mecanismos reguladores se diseñaron para nuevos cambios en los estilos de vida que fueron construyendo en la conciencia de la colectividad una nueva forma de cuidado de sí.

Así, la promoción de la salud se instauró como práctica comportamental en este campo, su intervención relacionó promoción-educación sobre los principales problemas de salud y sobre los métodos de prevención correspondientes:

La promoción de la alimentación y de los nutrientes adecuados, el abastecimiento adecuado de agua potable y el saneamiento básico, la asistencia materno-infantil, incluyendo la planificación familiar, la inmunización contra las principales enfermedades infecciosas, la prevención y lucha contra las enfermedades endémicas locales; el tratamiento apropiado de las enfermedades y traumatismos comunes y la disponibilidad de medicamentos esenciales (Tobar, 2001, p. 2).

Se reconoce en esta discusión la fuerte influencia de los organismos internacionales en la creación, difusión y legitimación de los modelos de salud a escala mundial y en particular para América Latina, que, apropiados en Colombia, inscribieron las políticas de salud que regirían en el país y actuaron como agentes catalizadores para vincular la salud y la educación como tarea de desarrollo que debían trazar los países miembros.

Para el caso colombiano, el camino de la salud-enfermedad a mediados del siglo XX, fuertemente influenciado por varios organismos norteamericanos como la fundación Rockefeller, la cooperación Técnica Norteamericana, delimitó el camino a seguir para combatir las enfermedades, enraizado en el discurso de la modernización y el mercado mundial. No obstante tratarse de un periodo en el que falta historiar con cuidado la práctica pedagógica en el escenario de la salud, las orientaciones impresas por las nuevas propuestas administrativas del sector salud y de las agencias internacionales impulsaron la creación de modelos sanitarios adoptados por el gobierno nacional, que establecieron como prioridades: "la orientación de las políticas y los planes sanitarios y en la formación del recurso humano en salud pública" (Eslava,1998, p. 107). 
Modernizar significaba "dotar de herramientas administrativas, políticas y financieras al Estado para intervenir y dirigir la economía por la senda del desarrollo económico y social" (OPS, 2002, p. 204). El paso de un Sistema Nacional de salud, como opción de integrar las acciones de protección de la salud, prevención de la enfermedad, curación y rehabilitación, a un sistema de Seguridad Social en Salud con la Ley 100 de 1993 pretendió hacer más eficiente el uso de los recursos y obtener mayor rendimiento; la pretensión: ampliar la cobertura para incorporar a toda la población bajo las estrategias de promoción de la salud.

En consecuencia, se hizo clara la intención de planificar los recursos humanos y se convirtió en la puerta de entrada para articular promoción con educación, que materializada en la Carta de Ottawa, se destacó la necesidad de desarrollar un máximo de potencial en salud y se estableció una relación estrecha entre paz, educación, salud, vivienda, alimentación ingreso, ecosistema, justicia social y equidad; "las habilidades y capacidades de los individuos, las condiciones sociales, ambientales y económicas serian los puntos claves de trabajo de los países" (OMS, 1986).

Clara intervención en el campo de la salud en su punto de cruce con la educación; aquí se entrevé la necesidad de una recontextualización para los dos campos, la cual fue materializada y apropiada para el campo de la salud en técnicas que mutaron de la intervención focalizada del control de las enfermedades, para la primera mitad el siglo XX, a la configuración de un modo de vida saludable, para la segunda. La emergencia de nuevos sujetos, disciplinas e institucionalidades permitió la reconfiguración de otra manera de pensar para replantear el problema de salud-enfermedad como una manera de cuidado de sí que tomó forma de auto gobierno.

\section{Promoción y educación en salud: un asunto de gubernamentalidad}

En continuidad con la línea de análisis, es preciso señalar que hoy la educación en salud (que lleva una clara acción pedagógica) es estudiada y apropiada a partir del concepto de promoción de la salud, y su pretensión es mostrar una dinámica y compleja visión de las relaciones salud-enfermedad que, al ser historiadas, despliegan diversos factores que la determinan y condicionan. Durante la primera mitad del siglo $X X$, la educación en salud es Ilamada educación sanitaria, donde la prevención y la profilaxis predominan a partir del disciplinamiento de cuerpos y mentes para lograr un cambio de comportamiento en el sujeto; para la segunda mitad del siglo XX, se circunscribe la promoción como comportamiento enunciado desde varios niveles (social, político, económico y cultural), donde la educación, dirigida a una u otra finalidad, toma la representación de promotora y terapéutica. 
Una nueva vertiente se abre para la práctica pedagógica cuando se encuentra operando al interior y exterior del campo de salud: la promoción de la salud, como saber y como práctica, transcurre en lo cotidiano en condiciones concretas, bajo las cuales, a través de la educación, pretende la conversión cultural hacia un saber de salud que, naturalizado y apropiado como deseable, traspasa todo el tejido social, pasando por la escuela, la familia, el hospital, el trabajo, etc.

Para los años cuarenta y cincuenta, la educación sanitaria consistió en instruir a las gentes en materia de higiene, de tal forma que se aplicaran los conocimientos adquiridos al perfeccionamiento de su salud, asunto referido por Grout (1948), citado por Salleras (1990, p. 55), "la educación sanitaria sería el procedimiento que entrañaba traducir los conocimientos acumulados acerca de la salud en normas de comportamiento adecuadas individual o comunitariamente, valiéndose para ello de la educación"; posteriormente, Gilbert (1959) afirmó que "la educación sanitaria pretendía inculcar actitudes sanas y formar una mentalidad de salud, apoyada en sólidas convicciones, como guía para elegir las reglas higiénicas de vida" (p. 55).

A partir de estos principios, la educación en salud durante la segunda mitad del siglo XX se concentró en los temas de enseñanza y enfatizó en los procesos de fortalecimiento de la autonomía; comportamiento, mentalidad y conducta que se relacionaron con la nueva salud pública de la promoción y, en este sentido, la OMS (2008) planteó: "no solamente los más pobres son quienes tienen mayor predisposición a mala salud, sino también quienes tienen menor nivel de educación, y ello no solo abarca nivel de escolarización, sino conocimiento en educación en salud".

En nombre de la promoción de la salud se justifican y legitiman reglas y normas que instalan las fuerzas del bien para constituir un régimen de verdad dirigido no a problemas sanitarios, sino a estilos de vida, cuyo propósito es administrar las conductas de los individuos actuando sobre sus posibilidades de acción a manera de concientización; paz, educación y salud es el eslogan concertado por la promoción para incidir y hacer posible ser apropiada. Parafraseando a Foucault (1977): se percibe la configuración de un poder de gubernamentalidad operada a partir de la medicalización de los comportamientos, conductas y discursos.

Los cambios operados en el concepto de salud en la nueva salud pública hablan de la promoción como un modelo para "mejores prácticas", donde se consideran altamente significativos los 
aspectos que definen: el problema/ desafío, los objetivos y resultados esperados, la población objetivo, los supuestos-valores-creencias, las teorías, la evidencia, el ámbito ético, la metodología, las formas de evaluación. Todo esto constituye el centro de reflexión y, más aún, una cantidad ilimitada de prácticas interiorizadas como patrones de conducta por la mayoría de la población.

Sin duda, la salud apropió el comportamiento y la conducta como metodología que facilitaría las relaciones con la educación; nos preguntamos entonces por la pluralidad de desarrollos que tuvo lugar en las actividades dirigidas a la transformación de los comportamientos de los individuos. Para Sutherland y Fulton (1992):

Tales actividades estarían enfocadas en estilos de vida y localizados en el seno de las familias, es decir, en las culturas de las comunidades en que se encuentran. Los programas y actividades de promoción en salud tienden a concentrarse en componentes educativos relacionados, en primera instancia, con riesgos comportamentales cambiables, que se encontrarían, por lo menos en gran parte, sobre el control de los propios individuos (p. 5).
En tal sentido, la promoción en salud ahora se presenta como "(...) el camino para lograr el respeto y las garantías de la protección y el derecho a la salud de todos y cada una de las personas del mundo, integrando la relación con los determinantes de salud"14 OPS (2007). La promoción de la salud se muestra como la estrategia, que en nuestro enfoque se constituye en un dispositivo múltiple, capaz de medirse desde ópticas objetivas y subjetivas para construir ciudadanos para la salud, y para ello la educación en salud se instaura en la herramienta articuladora entre el camino y la práctica.

Hay un nuevo poder que se ocupa de la vida a partir de la apropiación que el sujeto y en general la población hizo al concepto de promoción de la salud; este se relacionó directamente con el deseo de un comportamiento que llevara al individuo a mejorar sus condiciones de vida, que, sujeto a la categoría estilos de vida, emprendió la naturalización y el interés colectivo

14 Los determinantes de salud, según Lalonde ministro de Sanidad de Canadá, son: "Estilo de vida: es el que más influye en la salud y el más modificable; biología humana: se refiere a la herencia genética, que no suele ser modificable actualmente con la tecnología médica disponible; sistema sanitario: es el determinante de salud que más recursos económicos recibe para cuidar la salud de la población, al menos en los países desarrollados, y medio ambiente: se refiere a cualquier contaminación producida en el aire, suelo o agua que afecta la salud de los individuos, tanto si la contaminación es biológica, física, química o la llamada contaminación sociocultural y psicosocial, en la que incluiríamos la dependencia, violencia, estrés, competitividad, etc.". Recuperado de http://www.profesorenlinea.cl/Ciencias/SaludyEnfermedad.htm 
por la medicalización de la población, en tanto que cada individuo perfectamente diferente ingresa dentro del mismo deseo: ser saludable. Se instaura aquí una relación de inmanencia que reclama por la implementación de prácticas y comportamientos que permiten apropiarlo y reproducirlo a partir de un ejercicio oculto del poder ${ }^{15}$.

Como cierre de esta última parte, es importante indicar que la potencia de la educación en salud, en la clara alianza con la educación, pasó de ser un proyecto individual, en la primera mitad del siglo $X X$, a constituirse, durante la segunda mitad del siglo $X X$, en estructura y proceso social que abarcaba el medio ambiente físico y social, en tanto que los factores económicos como recursos, accesos a agua potable, vivienda y víveres se constituyeron en aspectos importantes y decisivos que la educación en salud debía tomar en cuenta para sus planteamientos de intervención. De esa manera, la educación en salud se planteó como un proceso social y político que se tornó imprescindible y se constituyó como elemento básico de la modernidad.

Explorar el vínculo entre las prácticas de saber y de poder como formas políticas sociales de conducir a los individuos en cuanto sujetos éticos, políticos y morales es un asunto que merece ser historiado para indagar a partir de la interioridad y exterioridad de un análisis discursivo la potencia que tuvo la alianza salud-educación como proyecto político-social-cultural en nuestro país.

\section{Conclusiones}

Se abre para la pedagogía un nuevo campo de discusión: la salud, pero, más que un debate pedagógico, es una discusión formulada a partir de su practicidad en las nuevas decibilidades, en cuanto noción de gubernamentalidad que proclama por la conversión de un sujeto, centrado en una economía de mercado globalizada.

La pedagogía, manipulada y enrarecida, se interroga por la potencia que tiene en la conversión de los sujetos; orienta sus condiciones de enseñanza a la instrucción de reglas y preceptos médicos, mediados por un saber científico. Aquí el maestro

15 Desde esta mirada, resulta importante seguir la pista que Foucault propone en su estudio de gubernamentalidad, cuando extendió su significado no solo a las prácticas gubernamentales del liberalismo o neoliberalismo, sino a la forma como se conduce la conducta de los hombres a partir de la "naturalización" y el "deseo" como formas de gobernamiento (2004, p. 156). 
adquiere otros rostros que van más allá de la escuela y se convierte en un educador sanitario, para regular no solo el cuerpo, sino la vida en una sociedad incluyente, marcada por los procesos de modernización de las sociedades capitalistas.

La práctica pedagógica en los dos escenarios de reflexión: salud y educación se apropia y moldea a partir de hechos históricos que definieron cada época; durante la primera mitad del siglo XX, en un sujeto unido a la categoría de progreso, y en la segunda mitad, unido a la categoría de desarrollo. Dos campos de intervención, un interés común, que, fundamentado en un sistema de homogenización, bajo efectos de un poder individualizante $y$ totalizante, es regulado, conducido y controlado a partir de una práctica que definió las necesidades de la población.

La democratización de la salud emerge como un objetivo primordial en esta nueva mirada, ello implica que el conocimiento, en general, y el conocimiento científico, en particular, serían los factores que facultarían a las personas para cambiar su realidad. Esta concepción se planteó como la nueva salud pública, con la que se transformó la idea de la relación con la salud-enfermedad y la higiene. De esa manera, la educación sanitaria amplió su perspectiva al dejar de estar enfocada, principalmente, en los pobres y las clases obreras, y buscó llegar a toda la población con sus técnicas de cuidado de sí y autorresponsabilidad, desplazando a la enfermedad por la salud; y para ello, se valió de la estrategia de promoción de salud, que actuó como dispositivo de control social, en tanto que vinculó nuevos actores diferentes al del sector salud.

El cuidado de sí y de los otros, como parte de la formulación de enunciados, invita a la reflexión más allá de un resultado y un deseo, de lo evidente y natural, sobresalta la sospecha y el asombro para tomar distancia. Por eso, más que un debate disciplinar, se trata de la pregunta por un sujeto vinculado entre la racionalidad y el poder, sin que este perciba sus efectos, en palabras de Foucault (1992, p. 248):

Será necesario por eso
modificar, reducir los
estados morbosos, pro-
longar la vida, estimular
la natalidad. Pero, sobre
todo, habrá que preparar
mecanismos reguladores
que en una población
global, puedan determi-
nar un equilibrio, con-
servar una medida. En
suma, habrá que instalar
mecanismos de seguri-
dad en torno a lo que
haya de aleatorio en las
poblaciones de seres
vivientes.

Pedagogía y salud, como asuntos de intervención, se analizaron a partir de un sistema de relaciones, de condiciones de existencia y de posibilidad que determinaron un lugar y una práctica común para los campos, marcados por líneas de fuerza 
que se cruzan en la interioridad y exterioridad de los enunciados que la regulan. En términos de Foucault: "la gubernamentalidad es más bien una vía para acceder a la racionalización de la gestión de los individuos, a la historia de la racionalidad tal y como opera en las instituciones y en la conducta de la gente" (Jodar y Gómez, 2007, p. 385).

\section{Referencias bibliográficas}

Álvarez, A. (2010a). La historia de la práctica pedagógica: una opción teórico-política. Documento inédito. Universidad Pedagógica Nacional.

Álvarez, A. (2010b). El estatuto teórico de la pedagogía en la transición posmoderna. Documento inédito. Universidad Pedagógica Nacional.

Álvarez, A. (2010c). Aporte para un debate sobre el estatuto teórico de la pedagogía. Documento inédito. Universidad Pedagógica Nacional.Banco Mundial y la Educación. (2006). Bretton Woods, 20. Recuperado de http://www.brettonwoodsproject.org/art.shtml?x=528478

Bourdieu, P. (2010). Cuestiones de sociología, Madrid, Itsmo, (1987). "Habitus, code, codification", Actes de la Recherche en Sciences Sociales, núm. 64.

Christopher, A. (1996). Ensayos de historia de la salud en Colombia, 1920-1990. Bogotá: Instituto de Estudios Políticos y Relaciones Internacionales. Universidad Nacional de Colombia.

Czeresnia, D. (2009). O Conceito de saúde e a diferença entre promoção e prevenção. En: promoção da Saúde, conceitos, reflexoes e tendências. Editora Fiocruz.

De Miguel, M. (1994). La calidad de la educación y las variables de proceso y de producto. En Pérez, R. (Coord.), Calidad de vida en los centros educativos (265-284). Asturias: Centro Asociado de la Uned.

Del Valle, A. y Vega, V. (1995). La capacitación docente. Una práctica sin evaluación. Argentina: Ediciones Magisterio del Río de la Plata.

Díaz, M. (1995). Aproximaciones al campo intelectual de la educación. En Larrosa, J. (Ed.), Escuela, poder y subjetivación. Madrid: La Piqueta. 
Díaz, M. (1998). Pedagogía, discurso y poder. Recuperado de http:// www.pedagogica.edu.co/storage/rce/articulos/19_14ensa.pdf

Secretaria de Salud (2007). Epidemiología. Sistema Nacional de Vigilancia Epidemiológica. N. 7, Vol. 24, Semana 7.

Echeverry, A. (1997). Hacia una pedagogía de la subjetivación. Recuperado de virtual.ustadistancia.edu.co/mod/resource/view. php?id=53631

Echeverry, A. (2009). Campo conceptual de la pedagogía: una contribución. (Tesis inédita de doctorado). Universidad Pedagógica Nacional.

Escudero, J. (1984). La renovación pedagógica: algunas perspectivas teóricas y prácticas. En Escudero, J. y González, M., Algunos modelos teóricos y el papel del profesor. Madrid: Escuela Española.

Eslava, J. (1998). El influjo norteamericano en el desarrollo de la salud pública en Colombia. Revista Biomédica, 18(2), 101-109.

Foucault, M. (1977). Historia de la medicalización. Educación Médica y Salud, 11(1).

Foucault, M. (1992). Genealogía del racismo. Madrid: Ediciones La Piqueta.

Foucault, M. (2000). Defender la sociedad. Argentina: Fondo de Cultura Económico.

Foucault, M. (2004). Seguridad, territorio y población. Buenos Aires: Fondo de Cultura Económica.
Foucault, M. (2007). Arqueología del saber. México: Siglo XXI Editores.

Gajardo, M. (2009). La educación tras dos décadas de cambios. ¿Qué hemos aprendido? ¿Qué debemos transformar?, en Marchesi, A., Tedesco, J. y Coll, C. (coord.) (2009), Calidad, equidad y reforma de la enseñanza. Madrid: Santillana-OEI.

Gilbert, J. (1959). L'éducation sanitaire, théorie et pratique. París: Masson et Cie.

Gómez, M. (1998). La crítica al "cientificismo" y al "positivismo" en la obra del Grupo de Investigación Educativa de la Universidad Nacional: una mirada desde la teoría crítica. Revista de Educación y Pedagogía, 19/20(IX-X).

Gómez, R y González, E. (2004). Evaluación de la prevención de la enfermedad y la promoción de la salud: factores que deben considerarse. Revista Facultad Nacional de Salud Pública, 1(22).

Grout, R. (1948). Health teaching in schools, Filadelfia: Saunders Company.

Hard, M. y Negri, A (2000). Imperio. Cambridge. Harvard University Press.

Hernández, M. (2002). La salud fragmentada en Colombia, 19101946. Bogotá: Instituto de Salud Publica. Universidad Nacional de Colombia. 
Huberman, S. (1994). Cómo aprenden los que enseñan. La formación de los formadores. Buenos Aires: Aique Didáctica,

Jodar, F. y Gómez, L. (2007). Educación posdisciplinaria, formación de nuevas subjetividades y gubernamentabilidad neoliberal, herramientas conceptuales para un análisis presente. Revista Mexicana de Investigación Educativa, 32(12).

Leavel, S. y Clark, E. (1976). Medicina preventiva. Sao Paulo: McGraw-Hill.

Luhmann, N. (1996) Teoría de la sociedad y pedagogía. Barcelona: Paidós Educador.

Quiceno, H. (1998). De la pedagogía como ciencia a la pedagogía como acontecimiento. Revista Educación y Pedagogía, 19-20.

Márquez, J. y García, V. (2006). Poder y saber en la historia de la salud en Colombia. Medellín: Editorial Lealon.

Márquez, J. y García, V. (2006). Poder y saber en la historia de la salud en Colombia. Medellín: Editorial Lealon.

Martínez, A., Noguera, C., Castro, J. (2003), Currículo y modernización, cuatro décadas de educación en Colombia. Bogotá: Magisterio.

Mockus, A. y Charum, J. (1986). Conocimiento y comunicación en las ciencias y en la escuela. Educación y Cultura, 8, 22-29.

Muñoz, J. (2004). Desafíos para la educación popular en Colombia. Texto resultado del debate entre miembros del colectivo CEEDAL Colombia. Recuperado de www.sercoldes.org.co/ images/pdf/desafiosparalaeducacionpopular

Noguera, C. (2003). Los manuales de higiene: instrucciones para civilizar al pueblo. Revista Educación y Pedagogía, 34(XIV).

Noguera, C. (2013). La noción de gubernamentalidad y su utilidad para pensar la práctica pedagógica. Texto inédito.

OMS. (1983). Nuevos métodos de educación sanitaria en atención primaria de salud: Informe de un comité de Expertos de la OMS. Serie de Informes técnicos 690: Oficina Regional para Europa. OMS. Ginebra.

OMS. (1986). Primera Conferencia Internacional para la Promoción de la Salud. Ottawa, Canadá.

OMS. (2008). La atención primaria en salud: más necesaria que nunca. Informe sobre la salud en el mundo.

OPS. (2002). La OPS y el Estado colombiano: Cien años de historia 1990-2002. Bogotá. 
OPS. (2007). La renovación de la atención primaria en salud de las Américas. Documento de posición de la Organización Panamericana de la salud y la Organización Mundial de la salud. Revista Panamericana de la Salud, 2(21).

Quevedo, E. (1994). El humanismo de Mutis, proyección y vigencia. En José Celestino Mutis ante la higiene pública (98). Bogotá: Universidad de Nuestra Señora del Rosario.

Ratinoff. L. (1994). La crisis de la educación: el papel de las retóricas y el papel de las reformas. Revista Latinoamericana de Estudios Educativos, 3-4(XXIV).

Sáenz, O. (1997). Hacia una pedagogía de la subjetivación. Revista de Educación y Pedagogía, 19/20(IX-X).

Salleras L. (1990). Educación sanitaria. Principios. Métodos. Aplicaciones. Madrid: Díaz de Santos.

Sepilli, A. (1958). Educación sanitaria y salud pública. Perugia: Universidad de Perugia.

Sirotnik, K. (1994). La escuela como el centro del cambio. En Escudero, J. M. y González, M. T., Profesores y escuela. Madrid: Ediciones Pedagógicas.

Stramiello, C. (2010). Sistemas Educativos Modernos para América Latina. Revista Española de Educación Comparada, 16, 393-412.
Tamayo, A. (2006). El movimiento pedagógico en Colombia. Encuentro de los maestros con la pedagogía. Revista Histedbr On-line, 24, 102-113.

Tobar, F. (2001). Atención primaria en salud. Una alternativa para salir de la emergencia. Recuperado de http://www.isalud.com/ pdf/tobar2.pdf

UNESCO-OREALC. (1976). EVolución y situación actual de la educación en América Latina. Madrid: Santillana.

Valencia, W. (2008). La práctica pedagógica, un espacio de reflexión. Medellín: Universidad de Antioquia.

Vázquez, Z. (2003). La modernización educativa (1988-1994). México: El Colegio de México.

Yepes, F. et al. (1990). La salud en Colombia. Análisis Socio Histórico. Bogotá: Editorial Presencia. Zuluaga, O. (1999). Pedagogía e historia, historicidad de la pedagogía, la enseñanza un objeto del saber, Universidad de Antioquia, Editorial Universidad de Antioquia. Anthropos. Siglo del Hombre Editores

Zuluaga, O. (2005). Foucault: una lectura desde la práctica pedagógica. La pedagogía y la educación, pensar de otro modo. Pedagogía e historia. Bogotá: Universidad Pedagógica Nacional. 
